This is the author's version of an article that has been published in the ICDCM 2019 proceedings.

Changes were made to this version by the publisher prior to publication.

The final version of record is available at https://dx.doi.org/10.1109/ICDCM45535.2019.9232741

\title{
Decentralized Cost-Optimized Fuzzy Control of DC Microgrids
}

\author{
Elias Knöchelmann*, Alexander Männel ${ }^{\dagger}$, Björn Goetjes*, Svenja Tappe*, and Tobias Ortmaier* \\ ${ }^{*}$ Gottfried Wilhelm Leibniz Universität Hannover, Institute of Mechatronic Systems, Hannover, Germany \\ Email: Elias.Knoechelmann@imes.uni-hannover.de \\ ${ }^{\dagger}$ Bosch Rexroth AG, Lohr am Main, Germany
}

\begin{abstract}
This paper presents a novel method for designing a decentralized fuzzy controller for DC microgrids, aiming to reduce operating costs. Therefore, a classical fuzzy control is created for each power supplying grid user based on the voltage droop control. The control of the active rectifier is then extended with respect to the electric costs as an additional input. The input membership functions of this controller are in a next step optimized with a genetic algorithm, whereas two different approaches were used: first, only the membership function of the rectifier was optimized, secondly, the energy storage system was added to the optimization. The optimization was performed in terms of minimizing the operative costs of the DC microgrid. This method and its results were in the end compared with the optimized characteristic diagrams presented in [1]. These diagrams were an extension of the voltage droop curves and the optimization was also based on the operating costs of the grid. Results achieved with the new concept are as good as in the previously presented characteristic diagrams approach. In addition optimization time is reduced significantly (up to 50 times) and the definition of membership functions is more handy.

Index Terms-Energy Control, DC Microgrid, Fuzzy Control, Voltage Droop Control
\end{abstract}

\section{INTRODUCTION}

As stated in the Paris agreement of the 2015th UN Convention on Climate change, carbon emissions have to be reduced significantly. Therefore, the use of renewable energy has been increased. A major disadvantage of these, however, is the irregular, fluctuating power output. As a result, electricity prices become more variable and traded on stock exchanges, such as the Leipzig electricity exchange [2]. These variable prices are available for end consumers and companies [3]. Due to the variable pricing structure it is beneficial to use energy storages to support the grid and at the same time reduce the operating costs of the plant. The installation of storages in DC grids is easier than in AC grids, because only DC-DC converters are needed and no inverter. In addition, such DC microgrids can be used to distribute braking energy from one loads to others. So the efficiency can be increased [4].

This paper cnsequently examines such a DC microgrid more detailed. The exemplary grid is shown in Fig. 1 and consists of an active rectifier $\left(\mathrm{T}_{\mathrm{AC}}\right)$ which allows regenerative feeding into the main grid, a photovoltaic system $\left(\mathrm{T}_{\mathrm{Re}}\right)$ (represented by wind turbines), an energy storage $\left(\mathrm{T}_{\mathrm{Bat}}\right)$ and four robots $\left(\mathrm{T}_{1-4}\right)$ as standard industrial consumers.

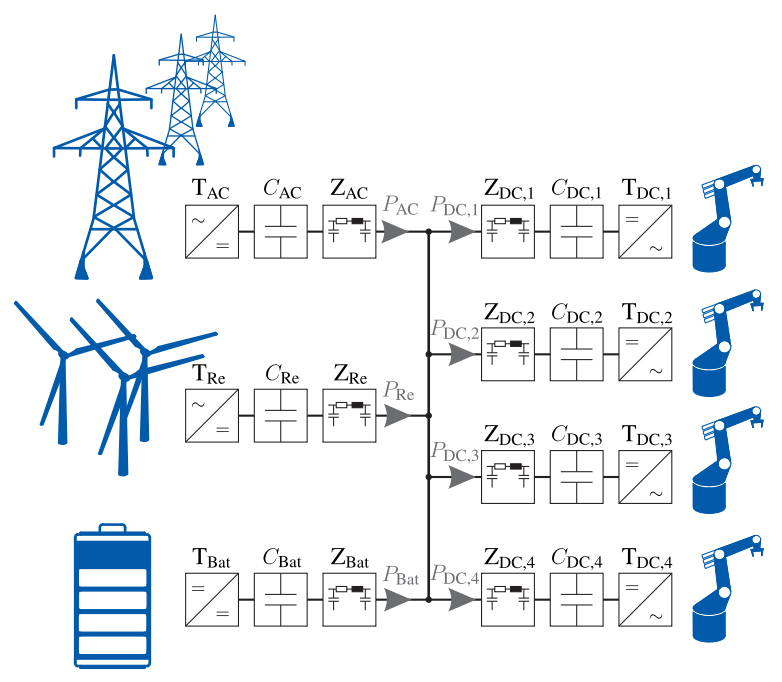

Fig. 1. Overview image of the investigated DC microgrid [1].

A large field of research is the control of such DC microgrids. The concepts can be roughly divided into decentralized and centralized approaches, see [5]. Central approaches have the advantage that the voltage band can be adhered to more precisely and load distribution between different suppliers can be better adjusted. However, a lot of communication is required and the network is not easily expandable, cf. [6]. Decentralized approaches, on the other hand, are easily expandable and require no real-time communication or no communication. An example of this is the voltage droop control, in which each active network participant is given a specific characteristic curve, given a desired relationship between voltage and current (see Fig 5). Depending on the voltage, the inverter provides the corresponding current. However, creating such characteristic curves has the disadvantage that they cannot be created intuitively, a more handy approach is to use fuzzy logic. The design of the controller is based on the experience of the engineer who designs this controller. Fuzzy control was used very early for the control of microgrids [7]. In [8] even a whole energy management with fuzzy controllers was implemented. However, the fuzzy controllers were not optimized but only heuristically set up. The principle structure of the control is shown in Fig. 2.

The generated characteristic curves behave very similar to

Copyright (c) 2019 IEEE. Personal use of this material is permitted. For any other purposes, permission must be obtained from the IEEE by emailing pubs-permissions@ieee.org. 
This is the author's version of an article that has been published in the ICDCM 2019 proceedings.

Changes were made to this version by the publisher prior to publication.

The final version of record is available at https://dx.doi.org/10.1109/ICDCM45535.2019.9232741

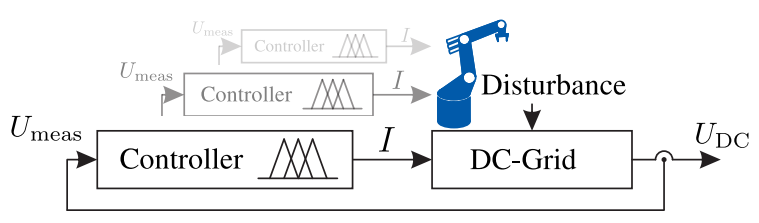

Fig. 2. Schematic representation of the fuzzy controller

the droop voltage curves and are compared with the normal characteristic curves in the following. The disadvantage of such curves is that they do not influence the load sharing of the grid [5].

The adaptive voltage droop control, in which the characteristic curves are adaptively readjusted, provides a workaround. In this case, the characteristic curve can also be adapted depending on the electricity price, so that the storage system is charged at a low and discharged at a high price. Another option without communication is to extend the dimension of the characteristic curves so that the current provided also depends on the electricity costs. This characteristic, as proposed in our previous work, diagrams were optimized offline based on the load cycle and then loaded onto the controller [1]. With this method, the advantages of the adaptation can be used without the need for additional communication and computing power. This paper takes up this idea and combines it with the previously described fuzzy control. In [8] a similar approach was presented, except the State of Charge (SOC) of the battery was chosen as an input instead of the electricity costs. In addition, in this work, the membership functions of Fuzzy controller of the storage system and active rectifier are optimized using a genetic algorithm, and not heuristically as in [8], provided that the fitness function includes the operating costs of the DC microgrid.

The article is organized as follows: In Section II, the layout of the DC microgrid is described and its modeling is presented. A short introduction in fuzzy control, as well as a comparison to classical control approaches is given in Section III. The structure of the used fuzzy controller is also presented. The optimization procedure is explained in Section IV. The results are discussed and compared in Section V. The paper closes with a conclusion in Section VI.

\section{SYSTEM DESCRIPTION AND MODELING}

For optimization of the fuzzy controller a model of the DC microgrid is needed. First, the structure of this is examined more closely. The investigated microgrid is shown in Fig. 1. It was assumed that a robotic cell is supplied with direct current and thus constitutes the load of this dc microgrid. Robots generate a lot of energy during braking movements. A large part (up to $17 \%$ [9]) of this energy is converted into heat due to the passive rectifiers of the robot control system. An expansion of the robot-internal dc circuit to several robots can, therefore, bring great energy savings. For this reason, four KUKA KR 16-2 robots were selected as loads within the DC microgrid. As shown in previous work [10], the energy consumption of each robot can be simulated/modeled with high accuracy.
As renewable energy source a photovoltaic system was added. For interruptibility power supply purposes a generic lithium-ion battery was included into the grid. The supply is realized as an active front end (AFE, active rectifier) converter to allow power regeneration into the main grid.

For the optimization, a randomized data set was generated for each robot covering twice 50 point to point movements. The power curves of these four robots were merged together with different pause lengths. The data for the photovoltaic system are measurements originated from a private facility. To increase the variance in the data, measurements were scaled down from 2.5 days to 2 hours. This also has the effect that a more complex test scenario has been created. The power curve used for optimization is shown in Fig. 3. The electricity price change was reduced from 15 minutes to 5 minutes for the same reason. Its change was modeled based on real electricity prices on the stock exchange in Germany [2]. In order to show that the optimization results are not only valid for the power curve used for optimization, a validation power curve fulfilling the same criteria was generated. The optimizations and comparisons in later parts were always based on the assumption that the energy storage is completely discharged at the start time in order to create better comparability.

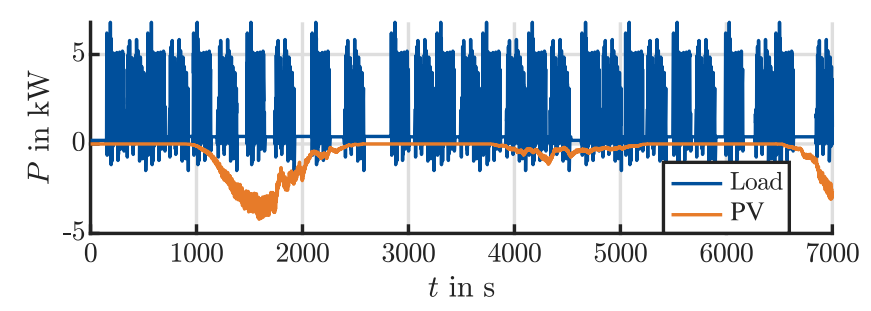

Fig. 3. Power curves used for optimization of fuzzy controller (this paper) as well as characteristic diagrams in [1].

\section{A. DC Grid Modeling}

The modeling of the DC Grid was done as proposed in [1] and will be explained here only briefly. Since the model of the DC network is invoked often in the optimization, a short runtime is mandatory. In order to fulfill this requirement, cable resistances were neglected based on their insignificance. Thus the intermediate circuit capacities can be combined into one capacity.

The general idea behind the DC grid modeling is that the energy $E$ stored in the electric field of a capacitor can be described by:

$$
E=\frac{1}{2} \cdot C \cdot U^{2}
$$

with $C$ being the capacity of a general capacitor and $U$ being the applied electrical voltage, synonymous with the grid voltage in this case. The controller output $I$, which depends on the DC gird voltage, needs to be converted to DC grid power by multiplying it with the DC grid voltage. The DC link power of active users can be calculated in this way, the DC grid power of passive users are either given as a load 
This is the author's version of an article that has been published in the ICDCM 2019 proceedings.

Changes were made to this version by the publisher prior to publication.

The final version of record is available at https://dx.doi.org/10.1109/ICDCM45535.2019.9232741

profile or can be measured. By multiplying the summed DC grid power $P_{\text {sum }}$ of each DC grid user by the time step $\Delta T$ used in the simulation the realized work $\Delta W_{\text {sum }}$ in time step $k$ is received

$$
\Delta W_{\text {sum }}(k)=P_{\text {sum }}(k) \cdot \Delta T \text {. }
$$

Under the assumption, that the realized work $\Delta W_{\text {sum }}$ is stored in the electrical field, Eqn. 1 can be rewritten as:

$$
U_{\mathrm{DC}}(k)=\sqrt{\frac{2}{C_{\mathrm{DC}}} \cdot\left|\Delta W_{\text {sum }}(k)\right|+U_{\mathrm{DC}}^{2}(k-1)} .
$$

Whereas $C_{\mathrm{DC}}$ is the DC link capacity and $U_{\mathrm{DC}}(k)$ is the resulting DC grid voltage for the time step $k$. With this equation the DC voltage can be calculated based on the consumer power.

The model was validated in [1] with the test setup described in [11]. The error between simulated and measured rectifier power for a 15 minute measurement is less than $4.5 \%$.

\section{FUZZY CONTROL}

Fuzzy control is based on fuzzy logic, which abandons the bivalent distinction between true and false and introduces fuzzy sets instead. The theory was developed by Lotfi Zadeh in 1965.

In fuzzy logic control engineering concepts can be developed with vague knowledge. These concepts have been established especially for unknown and non-linear (complex) controlled systems, since stable control loops can be implemented easily with a heuristic approach (i.e. fuzzy control) [12]. The effort required to implement these controllers can be significantly lower than with classical control concepts.

\section{A. Fuzzy control vs. classical control}

Fuzzy controllers are dynamic-free, non-linear, timeinvariant characteristic diagrams [12]. However, such diagrams can also be achieved using classical control methods, whereby the characteristic curve of the fuzzy controller cannot always cover the entire output range. This is due to the defuzzification method, because for example the boundary areas remain inaccessible via the center of gravity method [13]. A distinction can be made above all by the design methodology, clarity and vividness: Systematic design methods are available to the classical controller (e.g. p-controller), which enable a direct guarantee of stability and a specification of desired control criteria [12]. This is not available to the fuzzy controller, since it works according to heuristic methods and gives every developer a scope for interpretation. Therefore, the stability and the control behavior cannot be determined by trivial mathematical transformations, but have to be tested on the real model or in simulation.

Due to the similarities of both methods, there are no differences in robustness. The property can only be made tangible if it can become quantified as well. This means that a system model would be required to represent the maximum deviations between nominal and real distance.
An important criterion to compare control structures is interpretability and clarity. By means of the rule bases, the fuzzy controller proves to be manageable and easily interpretable. The behavior of the controller can be read directly from the rules. However, if the control requirements are very high (e.g. small deviation or fast controller response) and the number of rules become very high, the clarity decreases considerably. In the case of classical controllers, interpretations are still possible even with high complexity (e.g. on the basis of the state space representation or the determination of the system poles).

\section{B. Voltage Droop Curves with Fuzzy Logic}

In this section, an example of a characteristic curve for an active rectifier is created using fuzzy logic, based on the method proposed by Mamdani and Assilian [14]. First the corresponding membership functions are created. For industrial applications a guideline for the voltage level was given in [15]. $800 \mathrm{~V}$ is the upper and $400 \mathrm{~V}$ is the lower protective limit/boundary, these ranges may not be reached. The transient over (TueU) and the transient under voltage (TuU) are areas in which the DC voltage may only be present for a short time. In the stationary over (UeU) and under voltage (UU) range, the voltage may remain permanently, but the functionality of all devices is limited. The ideal voltage range is around $650 \mathrm{~V}$, for a larger control scope, this has been further divided into three ranges: lower normal voltage (NIU), normal voltage (NU) and upper normal voltage $(\mathrm{NrU})$. The associated membership functions are shown in Fig.4 Part I.
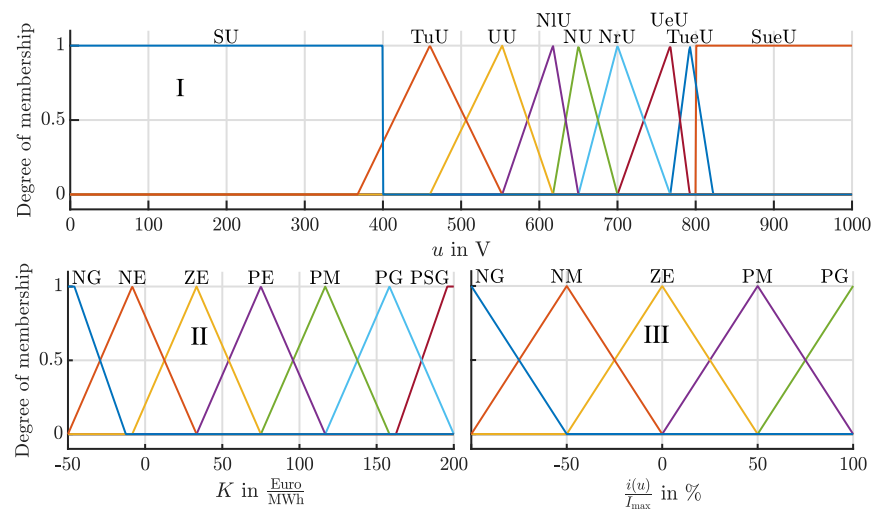

Fig. 4. Input membership functions of the voltage (I), the electric costs(II) and the output membership functions for the current (III) of the AFE and the storage.

The output of the controlled system is the current $I$ provided by the active rectifier or storage system. The associated membership functions are shown in Fig.4 Part III. For division optimal coverage (cf. [8]) was chosen, where the $x$-axis indicates the actual current related to the maximum current. NG stands for negative large, NM for negative medium, ZE for zero, PM for positive medium and PG for positive large.

The examplary fuzzy rules are shown in Tab. I . These were chosen intuitively, in a way that, e.g. , at UU the supplier must set NG, to achieve that the grid voltage is led to normal range. 
This is the author's version of an article that has been published in the ICDCM 2019 proceedings.

Changes were made to this version by the publisher prior to publication.

The final version of record is available at https://dx.doi.org/10.1109/ICDCM45535.2019.9232741

TABLE I

FUZZY CONTROL RULES FOR THE RECTIFIER

\begin{tabular}{|c|l|l|l|l|l|l|l|l|l|} 
voltage & SU & TuU & UU & NIU & NU & NrU & UeU & TueU & SueU \\
\hline output & NG & NG & NG & NM & ZE & PM & PG & PG & PG
\end{tabular}

From these membership functions and the given fuzzy rules the droop curves (blue) shown in Fig. 5 results. Optically the characteristics differ the droop curves, which were created according to [11], conventionally generated voltage droop curves. due to the decreasing of the gradient at $\pm 80 \%$, as well as the smaller plateaus in the battery curve. This, together with the steeper AFE curve, results in less oscillating around the guide voltage value.

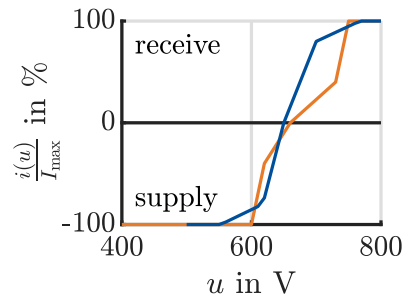

(a) Droop curves of the rectifier

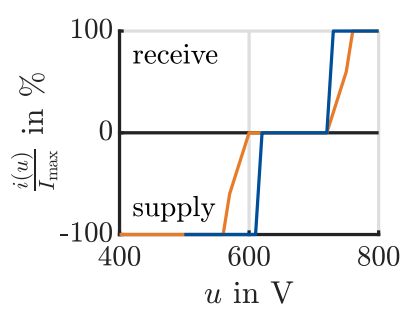

(b) Droop curves of the battery
Fig. 5. Droop curves for active grid users resulting from defuzzification in blue and in orange according to [11] conventionally generated voltage droop curves.

\section{Extension of the Fuzzy Control}

A second input must be defined for the fuzzy controller in order to take electricity costs into account. The corresponding membership functions are shown in Fig. 4 Part II, where NG stands for negative large, NE for negative/around zero, $\mathrm{ZE}$ for zero, PE positive small, PM positive medium, PG positive high and PSG for positive very high. The fuzzy rules for the active rectifier are shown in Tab. II. The cost allocation for the battery was reversed, since the battery has to be discharged at high power costs and charged at low costs. With the active rectifier, the situation is exactly the other way around. To increase the optimization possibilities, the normal voltage was not, as shown in Fig. 4, divided into 3 areas, it was divided into 5 areas. For simplicity's sake, these were simply referred to as N1-N5.

From these membership functions and the fuzzy rules result the characteristic diagrams shown in Fig. 6.

\section{OPTIMIZATION}

As stated in [16] two ways exist how to optimize fuzzy controllers. On the one hand the fuzzy rules can be optimized and on the other hand the membership functions. In [16] it was noted that the optimization of the fuzzy rules can lead to a "Bang-Bang" regulation. On the other hand the characteristic diagrams should follow certain laws: at low voltage all active grid users should feed the DC mircogrid, at high voltage all should feed back into the main grid. In
TABLE II

FUZZY CONTROL RULES FOR THE RECTIFIER INCLUDING ELECTRICITY COSTS

\begin{tabular}{|c|c|c|c|c|c|c|c|c|}
\hline \multirow{2}{*}{\multicolumn{2}{|c|}{ ouput }} & \multicolumn{7}{|c|}{ costs } \\
\hline & & $\mathrm{NG}$ & $\mathrm{NE}$ & $\mathrm{ZE}$ & $\mathrm{PE}$ & PM & PG & PSG \\
\hline \multirow{11}{*}{$\begin{array}{l}\mathscr{0} \\
\frac{\pi}{0} \\
\frac{\pi}{0}\end{array}$} & $\mathrm{SU}$ & $\mathrm{NG}$ & $\mathrm{NG}$ & $\mathrm{NG}$ & $\mathrm{NG}$ & $\mathrm{NG}$ & NG & $\mathrm{NG}$ \\
\hline & TuU & $\mathrm{NG}$ & $\mathrm{NG}$ & $\mathrm{NG}$ & $\mathrm{NG}$ & $\mathrm{NG}$ & $\mathrm{NG}$ & $\mathrm{NG}$ \\
\hline & UU & $\mathrm{NG}$ & $\mathrm{NG}$ & $\mathrm{NG}$ & $\mathrm{NG}$ & $\mathrm{NG}$ & NM & ZE \\
\hline & N1 & $\mathrm{NG}$ & NG & NG & NM & NM & $\mathrm{ZE}$ & PM \\
\hline & N2 & $\mathrm{NG}$ & NG & NM & NM & $\overline{Z E}$ & PM & $\overline{P M}$ \\
\hline & N3 & $\mathrm{NG}$ & $\mathrm{NM}$ & NM & $\mathrm{ZE}$ & PM & PM & PG \\
\hline & N4 & NM & $\mathrm{NM}$ & $\mathrm{ZE}$ & PM & PM & PG & PG \\
\hline & N5 & $\mathrm{NM}$ & $\mathrm{ZE}$ & PM & PM & PG & PG & $\mathrm{PG}$ \\
\hline & $\mathrm{UeU}$ & $\mathrm{ZE}$ & PM & PG & PG & PG & PG & PG \\
\hline & TueU & PG & PG & PG & PG & PG & PG & PG \\
\hline & SueU & PG & PG & PG & PG & $\mathrm{PG}$ & $\mathrm{PG}$ & $\mathrm{PG}$ \\
\hline
\end{tabular}
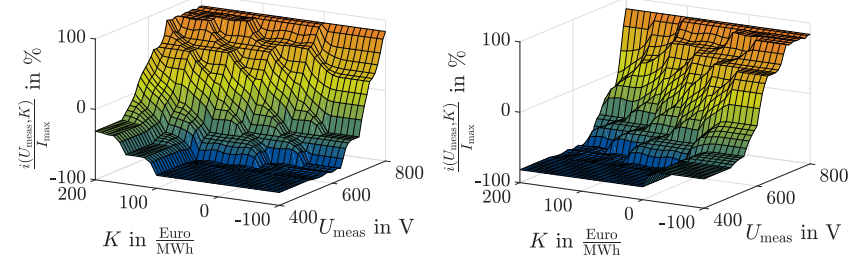

(a) Initial characteristic diagram for (b) Initial characteristic diagram for active rectifier control.
Fig. 6. Display of the initial characteristic diagrams resulting from defuzzification on the rules form Table II.

addition, the characteristic diagrams should be a monotonic (growing) function. In [1] a great effort was made to prevent this. Due to the predesigning of the fuzzy rules this post processing is not necessary, but by optimizing the fuzzy rules this advantage would be lost again. For this reason only the membership functions are optimized.

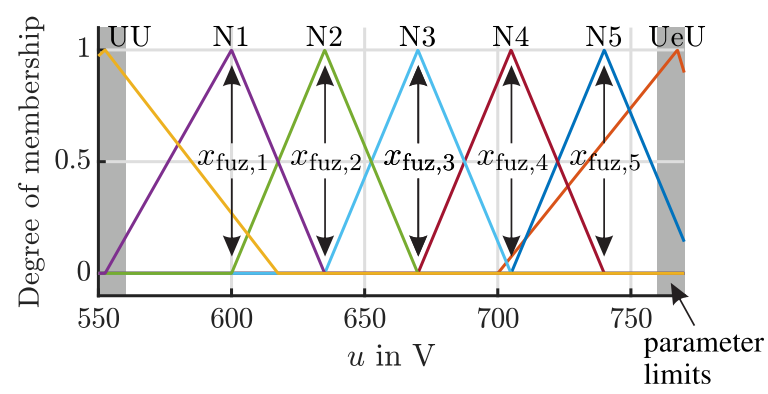

Fig. 7. Input membership functions of the voltage of the AFE and the storage in the range from 550 to $770 \mathrm{~V}$ to display the optimization parameters $x_{\mathrm{fuz}, i}$.

Fig. 7 shows the optimization parameters that describe the membership functions. Since the voltage of the DC microgrid should only be in the normal range, i.e. between $560 \mathrm{~V}$ and $760 \mathrm{~V}, \mathrm{~N} 1-\mathrm{N} 5$ was assumed to be optimizable. All other membership functions, of the voltage, are outside this range and are not changed. For the costs NE, ZE, PE, PM and PG are assumed to be optimizable. The parameter limits are $-50 \frac{\text { Euro }}{\mathrm{MWh}}$ and $200 \frac{\text { Euro }}{\mathrm{MWh}}$. Three parameters are required for 
This is the author's version of an article that has been published in the ICDCM 2019 proceedings.

Changes were made to this version by the publisher prior to publication.

The final version of record is available at https://dx.doi.org/10.1109/ICDCM45535.2019.9232741

each membership function: the beginning $x_{\mathrm{fuz}, i-1}$ the peak $x_{\mathrm{fuz}, i}$ and the end $x_{\mathrm{fuz}, i+1}$. Since an optimal coverage was chosen, the precede peak can be used for the beginning and the subsequent peak for the end. This results in the description of the membership functions:

$$
\Lambda(u)= \begin{cases}\frac{u-x_{\mathrm{fuz}, i-1}}{x_{\mathrm{fuz}, i-}-x_{\mathrm{fuz}, i-1}} & x_{\mathrm{fuz}, i-1} \leq u \leq x_{\mathrm{fuz}, i} \\ \frac{x_{\mathrm{fuz}, i+1}-u}{x_{\mathrm{fuz}, i+1}-x_{\mathrm{fuz}, i}} & x_{\mathrm{fuz}, i} \leq u \leq x_{\mathrm{fuz}, i+1} \\ 0 & \text { else. }\end{cases}
$$

This results in 5 optimization parameters for the cost and 5 for the voltage. An important limitation is that the membership functions must stay arranged in the given folder. The following constraint results for optimization:

$$
x_{\mathrm{fuz}, i-1}<x_{\mathrm{fuz}, i}<x_{\mathrm{fuz}, i+1} .
$$

According to [12] two types of parametrization are possible:

- Absolute parameterization: The peaks $x_{\mathrm{fuz}, i}$ of the triangle functions are given directly from the optimization algorithm $x_{\mathrm{GA}, i}$. A disadvantage here is that the required limits make it difficult to optimize parameters that lie within the defined range.

$$
x_{\mathrm{fuz}, i}=x_{\mathrm{GA}, i}
$$

- Relative parameterization: The peaks $x_{\mathrm{fuz}, i}$ of the triangle functions are sum of the preceding peak $x_{\mathrm{GA}, i-1}$ and the optimization result $x_{\mathrm{GA}, i}$. Here it is easier to keep the limits. However, changing the first one will change all peaks positions and therefore finding the optimum is challenging.

$$
x_{\mathrm{fuz}, i}=x_{\mathrm{GA}, i-1}+x_{\mathrm{GA}, i}
$$

As a result of the simulation evaluation, the optimization with absolute parameterization found the first valid parameter set later than with relative, but the final parameter set had lower costs. Therefore the absolute parameterization was used. In the following, two different optimizations are performed:

- first only the AFE fuzzy controller has been optimized, the storage fuzzy controller from Fig. 6b. remains unchanged.

- In the second optimization, both fuzzy controllers were optimized.

As cost function for the optimization the cost function presented in [1] is used. The total costs were standardized to monetary costs and $\zeta_{\text {tot }}$ are thus calculated as follows:

$$
\zeta_{\text {tot }}=K \cdot \int_{t_{0}}^{t_{\text {end }}} P_{\mathrm{AFE}} \mathrm{d} t+\frac{K_{\mathrm{inv}} \cdot E_{\mathrm{LI}}}{a \cdot \mathrm{e}^{b \cdot \mathrm{DOD}}}-K_{\text {end }} \cdot E_{\text {bat,stor }} .
$$

$P_{\mathrm{AFE}}$ is the converted rectifier power, $t_{0}$ is the point in time at which the electricity costs $K$ change and $t_{\text {end }}$ up to which the costs remain constant. Therefore the costs for regenerating and receiving energy from the main grid are described. The second part of the equation describes the costs of the lifetime change of the energy storage due to discharge and charging. Thereby $K_{\mathrm{inv}}$ is the investment cost for the energy storage, $E_{\mathrm{LI}}$ is the storable energy, $a$ and $b$ are the fitted parameters, that describe the relationship between depth of discharge (DOD) and the cycle reduction. Data on the discharge depth and lifetime were taken from [17]. The investment costs for lithium-ion batteries were set to $200 \frac{\text { Euro }}{\mathrm{MWh}}$ based on [18]. The last part takes the energy in the energy storage $E_{\text {bat,stor }}$ at the end of simulation into account. This energy will be evaluated with the last electricity price $K_{\text {end }}$.

\section{RESULTS}

In this section the created fuzzy controllers are evaluated based on the performance profile from Fig. 3 .

\section{A. Initial Fuzzy Control}

In Fig. 8 the upper plot shows the DC grid voltage in blue and the feed-in power of the active rectifier in orange. The left $y$-axis limit is equal to the power limit of the active rectifier (AFE) $(30 \mathrm{~kW})$ and the energy storage $(30 \mathrm{~kW})$, respectively. The axis label for the voltage is on the right $y$-axis. The plot underneath shows the power curves of the individual grid users. The electricity cost over time is plotted on the right $y$-axis. This design will remain the same for all subsequent plots.

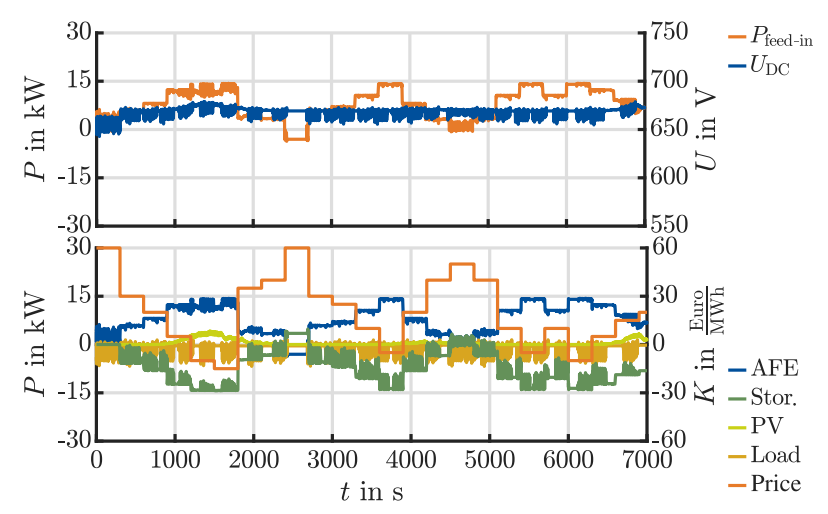

Fig. 8. Plot of the different characteristics of the simulated DC microgrid with the initial fuzzy controllers for rectifier and energy storage system from Fig. 6

The voltage in the upper part of Fig. 8 is within the predefined range and relative smooth. The power consumption and output of the active rectifier is far below the limits. In the lower plot it can be seen that the storage system and the AFE work in opposite directions. The cost of operating the DC grid for the given period of time $(7000 \mathrm{~s})$ is $4.54 €$. The cost is mainly due to switching between charging and discharging. This can be seen between 4000 and $5000 \mathrm{~s}$, where the storage power (green) crosses zero very often. Despite this, the costs are moderate compared to the droop voltage control from [1], which resulted in $15.89 €$. 
This is the author's version of an article that has been published in the ICDCM 2019 proceedings.

Changes were made to this version by the publisher prior to publication.

The final version of record is available at https://dx.doi.org/10.1109/ICDCM45535.2019.9232741

\section{B. Rectifier Optimized Fuzzy Control}

In Fig. 9, the fuzzy controller for the active rectifier, i.e. the position of the membership functions from Fig. 4, were optimized. The resulting characteristic diagram has a similar shape as Fig. 10.a and is not shown here for redundancy reasons. The voltage in the upper plot is not as smooth as the voltage in Fig. 8. In the lower area it can be seen that the storage system and the active rectifier work in opposite directions, but make even less use of the power limits (here $\max 13 \mathrm{kWh}$, compared to $15 \mathrm{kWh}$ in Fig. 8). However, the operating costs were reduced to $0.33 €$ compared to the initial fuzzy controller. This is because the storage system no longer switches rapidly between charging and discharging.

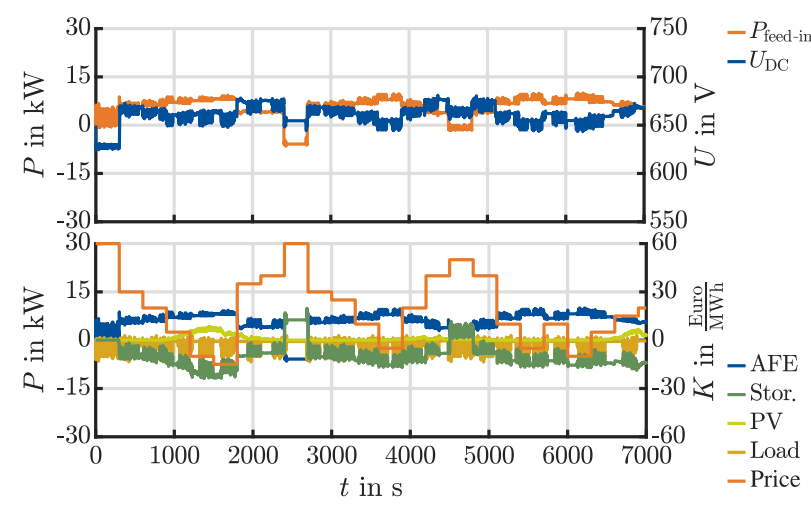

Fig. 9. Plot of the different characteristics of the simulated DC microgrid with the rectifier optimized fuzzy controller

\section{Rectifier and Storage System Optimized Fuzzy Control}

In this section the membership functions of the active rectifier and the energy storage system were optimized. The resulting characteristic diagrams are shown in Fig. 10. It can be seen that steep edges were created at a electric cost value of zero. This ensures that charge and discharge states have different behavior, i.e. at the same electricity costs, the voltage at which the storage device does provide no current is very different. Thus fast changing between the two states is prevented.
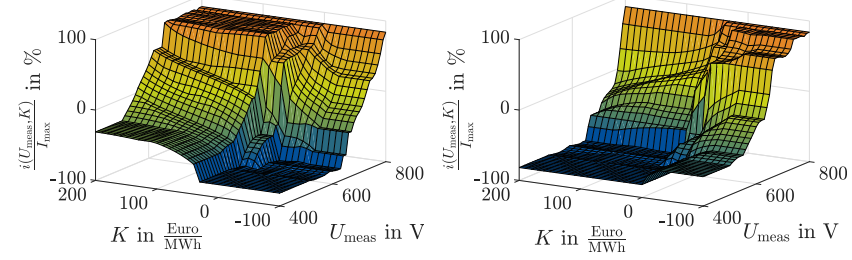

(a) Optimized characteristic diagram (b) Optimized characteristic diagram for active rectier control. for energy storage control system.

Fig. 10. Display of the active rectifier and energy storage optimized characteristic diagrams resulting from defuzzification.

In Fig. 11 the effects of the optimized control on the test scenario are shown. The voltage in the upper plot is even less smooth than in the preceding scenario. The voltage drops occur when the energy storage is empty. It can no longer transfer any energy to the microgrid, as a result the voltage drops. Since the electricity costs are high, the grid is only supplied by the AFE in an emergency, to reduce the energy in the DC microgrid and, as a result, the operating costs. The operating costs amount to $0.25 €$.

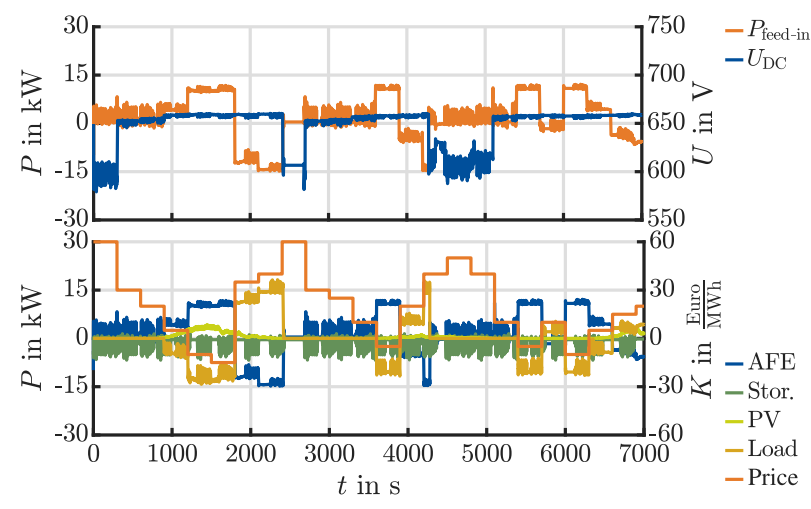

Fig. 11. Plot of the different characteristics of the simulated DC microgrid with the rectifier and energy storage optimized fuzzy controller

\section{Comparison of the different control concepts}

In this section, the different approaches are compared based on their operating costs and the duration of the optimization. The optimized characteristic diagrams from [1] serve as comparison. The simulated course of the microgrid with these diagrams is shown in Fig.12. Compared to the other courses (Fig. 8, 9 \& 11 ), it is immediately noticeable that the performance limits of the AFE and the energy storage system were exploited. With fuzzy control, the limit values cannot be reached (see section III) and the gradient of the resulting characteristic diagrams is restricted by the fuzzy rule. This has an influence on the operating costs, which are even negative (see Tab. III).

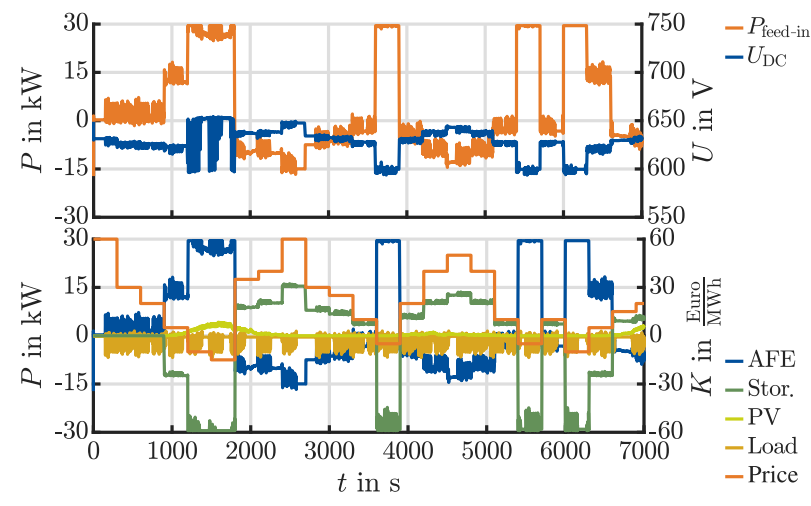

Fig. 12. Plot of the different characteristics of the simulated microgrid with the finalized characteristic diagrams from [1]

In Tab. III all operating costs are summarized, where the 1st opt. fuzzy CD is the optimization of the AFE membership 
This is the author's version of an article that has been published in the ICDCM 2019 proceedings.

Changes were made to this version by the publisher prior to publication.

The final version of record is available at https://dx.doi.org/10.1109/ICDCM45535.2019.9232741

functions (section V.B.) and the 2nd is the optimization of the AFE and storage membership functions (section V.C.). For the validation of the results a validation performance course was used, which was already introduced in [1]. It is particularly noticeable that the first optimization approach generates large operating costs. This can again be explained by the fast switching between loading and unloading. The second optimization does not overfit to the optimization process, since the operating costs remain in similar value range. Both optimizations, however, do not approach the savings presented in [1], since the degrees of the freedom within the optimization in [1] were much larger. However, this has an effect on the optimization duration. With the postprocessing of the characteristic diagrams the optimization of these lasted approx. $50 \mathrm{~h}$. Sorely optimizing the rectifier took about one hour, whereas optimizing rectifier as well as energy storage system took about $10 \mathrm{hrs}$. Thus, the first optimization is suitable for an online optimization due to its 'short' runtime. With online optimization, a new characteristic map should not be calculated for every change in the load curves. Only if the operational behaviour of the DC microgrid deteriorates (e.g. too high costs, too low voltage) could a new map be optimized based on measured data. A big advantage is also that no further postprocessing is necessary, even if the reason for it, the fixed fuzzy rules, prevents as satisfying results as shown in [1].

TABLE III

COSTS FOR ONE MODEL RUN WITH DIFFERENT CHARACTERISTIC DIAGRAMS (CD)

\begin{tabular}{|c|c|c|c|c|}
\hline applied method & $\begin{array}{c}\text { initial } \\
\text { fuzzy CD }\end{array}$ & $\begin{array}{c}\text { 1st opt. } \\
\text { fuzzy CD }\end{array}$ & $\begin{array}{c}\text { 2nd opt. } \\
\text { fuzzy CD }\end{array}$ & $\begin{array}{c}\text { opt. } \\
\text { CD [1] }\end{array}$ \\
\hline $\begin{array}{c}\text { optimization course } \\
\text { of performance }\end{array}$ & $4.54 €$ & $0.33 €$ & $0.26 €$ & -0.29 \\
\hline $\begin{array}{c}\text { validation course } \\
\text { of performance }\end{array}$ & $13.39 €$ & $6.41 €$ & $0.25 €$ & $-0.15 €$ \\
\hline
\end{tabular}

\section{CONCLUSION}

In this article a fuzzy control for DC microgrids was presented. First droop voltage curves were created by fuzzy logic. These were then extended by a further input, the electric costs, to characteristic diagrams. In the following step it was examined, which possibilities for optimization are promising for success. It was decided not to optimize the fuzzy rules but only the input membership functions. By the given fuzzy rules a monotonous map can be created, which would have to be created afterwards if the rules were optimized. Two optimizations were performed: first only the membership function of the rectifier was optimized, second the membership function of the rectifier and the energy storage system were both optimized. The optimization results were compared with each other and with the results from [1]. It was shown that optimizing only the membership function leads to fast optimization times. The method used from [1] can lower the operating costs a little further, but with more severe additional time effort. This method is, therefore, a successful alternative, if the time is the limiting factor, since post processing is no longer necessary and the design is thus simplified.

\section{ACKNOWLEDGMENT}

This project was part of the DC-INDUSTRIE research project, funded by the German Federal ministry of Economic Affairs and Energy (BMWi).

\section{REFERENCES}

[1] E. Knöchelmann, A. Männel, S. Tappe, and T. Ortmaier, "Cost-optimized control of dc microgrids based on characteristic diagrams," in 2019 IEEE International Conference on Industrial Technology (ICIT), 2019, pp. $1685-1691$

[2] European Energy Exchange, "Leipzig electricity stock exchange," 2018 [Online]. Available: https://www.eex.com/de/

[3] Next Kraftwerk, "Variable electricity tariffs for industry \& commerce," 2018. [Online]. Available: https://www.next-kraftwerke.de/virtuelleskraftwerk/stromverbraucher/variabler-stromtarif

[4] M. Pellicciari, A. Avotins, K. Bengtsson, G. Berselli, N. Bey, B. Lennartson, and D. Meike, "Areus-innovative hardware and software for sustainable industrial robotics," in Automation Science and Engineering (CASE), 2015 IEEE International Conference on, 2015, pp. 1325-1332.

[5] T. Dragicevic, X. Lu, J. Vasquez, and J. Guerrero, "Dc microgrids-part i: A review of control strategies and stabilization techniques," IEEE Transactions on Power Electronics, p. 1, 2015.

[6] L. Meng, E. R. Sanseverino, A. Luna, T. Dragicevic, J. C. Vasquez, and J. M. Guerrero, "Microgrid supervisory controllers and energy management systems: A literature review," Renewable and Sustainable Energy Reviews, vol. 60, pp. 1263-1273, 2016.

[7] T. Funabashi, G. Fujita, K. Koyanagi, and R. Yokoyama, "Field tests of a microgrid control system," in Proceedings of the 41st International Universities Power Engineering Conference. Piscataway, NJ: IEEE Operations Center, 2006, pp. 232-236.

[8] Y.-K. Chen, Y.-C. Wu, C.-C. Song, and Y.-S. Chen, "Design and implementation of energy management system with fuzzy control for $\mathrm{dc}$ microgrid systems," IEEE Transactions on Power Electronics, vol. 28, no. 4, pp. 1563-1570, 2013.

[9] E. Knöchelmann, J. Kotlarski, T. Böhm, S. Tappe, and T. Ortmaier, "Potential of energy storage systems for industrial robots," 4th $\mathrm{MHI}$ Colloquium, 2019.

[10] K. Eggers, E. Knöchelmann, S. Tappe, and T. Ortmaier, "Modeling and experimental validation of the influence of robot temperature on its energy consumption," vol. 2018, 2018.

[11] A. Männel, S. Tappe, E. Knöchelmann, and T. Ortmaier, "Investigation on an ac grid failure handling of industrial dc micro grids with an energy storage," in 2019 IEEE International Conference on Industrial Technology (ICIT), 2019, pp. 1710-1716.

[12] K. Michels, F. Klawonn, R. Kruse, and A. Nürnberger, Fuzzy Control: Fundamentals, Stability and Design of Fuzzy Controllers, ser. Studies in fuzziness and soft computing. Berlin, Heidelberg: Springer, 2006, vol. 200.

[13] D. Driankov, H. Hellendoorn, and M. Reinfrank, An Introduction to Fuzzy Control. Berlin, Heidelberg and s.l.: Springer Berlin Heidelberg, 1993.

[14] E. H. Mamdani and S. Assilian, "An experiment in linguistic synthesis with a fuzzy logic controller," International Journal of Man-Machine Studies, vol. 7, no. 1, pp. 1-13, 1975.

[15] DC-INDUSTRIE, "A concept for a dc grid in industrial production: Dc-industrie - intelligentes offenes dc-netz in der industrie für hocheffiziente systemlösungen mit elektrischen antrieben," 2018. [Online]. Available: https://dcindustrie.zvei.org/fileadmin/DC-Industrie/Praesentationen/180202_DCINDUSTRIE_DC_grid_concept_A04w.pdf

[16] P. Thrift, "Fuzzy logic synthrsis with genetic algorithms," 1991.

[17] I. Stadler, Energiespeicher: Bedarf, technologien, integration. Berlin: Springer Vieweg, 2014

[18] Horváth \& Partners, "Global price development for lithium-ion batteries from 2013 to 2020," 04.2016. [Online]. Available: https://de.statista.com/statistik/daten/studie/534429/umfrage/weltweitepreise-fuer-lithium-ionen-akkus/ 